\title{
High Resolution Spectroscopic Campaigns to Support Spacecraft Observations of Comets with Small Telescopes
}

\author{
Walter M. Harris
}

Received: 28 January 2009/Accepted: 28 May 2009/Published online: 16 June 2009

(C) The Author(s) 2009. This article is published with open access at Springerlink.com

\begin{abstract}
Ground based observations of comets obtained in support of spacecraft missions and space telescopes have provided critical supporting context that greatly enhances the value of the combined dataset. Major areas of ground-based contribution include providing unique instrumental capabilities and an increased temporal or global perspective on the system under study. This paper describes a decades long program of supporting cometary observations focused on high resolving power measurements of ions and atomic/ molecular radicals in the coma. The instrumentation is described, along with the species under study and the results from a large campaign to study comet C/1995O1 (Hale-Bopp).
\end{abstract}

Keywords Comets $\cdot$ High resolution spectroscopy $\cdot$ Atomic and molecular spectroscopy

\section{Introduction}

Observations from above the Earth's atmosphere have provided an increasingly rich and new perspective on comets during the previous 30 years. Orbiting observatories now provide access to both neutral and ion coma constituents with resonances in the X-Ray (e.g. ROSAT, Chandra), UV (e.g. IUE, FUSE, HST), IR (e.g. Spitzer, ISO, ODIN), and millimeter (e.g. SWAS) spectral ranges where the Earth's atmosphere is opaque. Comet encounter missions have further improved our understanding of nuclei and the inner coma through a combination of passive sensing (e.g. Vega, Giotto), sample return (e.g. Stardust), and direct interaction (e.g. Deep Impact, Rosetta). The addition of these capabilities has greatly expanded our ability to observe molecular parents, the detailed structure of the nucleus, and the evolution of outgassing at locations near the Sun.

While space observations have opened many new areas of cometary research, they cannot, in and of themselves, take the place of a robust ground-based program of study. Ground based support typically provides significant benefit in three clear areas. First, they are able to give temporal context, especially for targets (like Comets) that change 
significantly during the course of a mission or relative to a single space telescope observation. This is particularly important for fly-by missions where the time with the target is short. In this regard, observations made from smaller telescopes can be especially helpful, because they are generally undersubscribed compared to larger facilities and can thus be scheduled for either greater or more precise temporal coverage. A second area of contribution is in providing a technical capability or observational perspective that is complementary to the remote spacecraft. By their nature, space-observing facilities are limited in their capability to smaller, more generalized instrumentation. Many important techniques (e.g. high resolving power spectroscopy, single species narrow band imaging) are difficult to incorporate in a space platform, especially a remote probe. Finally, ground based programs provide a key historical context, since many diagnostic features of the coma have databases extending back more than 50-100 years. The value of such complementary data has driven the organization of many large campaigns (e.g. Meech et al. 2005) that have been operated in consort with space missions of various types.

A particular area where ground based capabilities are valuable is observations where high spectral resolving power is required. This capability has not been incorporated on any in situ comet missions flown to date, primarily due to the large size of echelle spectrometers and their very low étendue when used with small collecting area telescopes. Space telescopes (HST-STIS, IUE, FUSE) have had high resolving power modes with large collecting areas. However, they typically use small apertures that capture only small fields of view (FOV), making them a poor match to the large angular scale and low surface brightness of coma emissions. This is a limitation shared by similar instruments used at ground-based telescopes, but can be partially offset by larger collecting areas and longer duty cycles. Better sensitivity over the full coma can be obtained using wide field, high étendue instruments (e.g. FTS), hyperspectral imagers, or integral field spectrometers, but these technologies are still rarely implemented in space flight. However such instruments are readily available to ground based observers.

Beginning with the apparition of comet C/1973 E1 (Kohoutek) observers at the University of Wisconsin, in subsequent collaboration with teams from the Goddard Spaceflight Center, the University of Washington, and the University of California, Davis, have conducted synoptic observations of comets using interferometric instrumentation focused on obtaining high spectral resolving power measurements of ion and neutral emission line features. Over the next 35 years this program has grown to include several different classes of high resolving power instruments, used primarily at facilities with telescope apertures of $<4 \mathrm{~m}$ that permit the implementation of long campaigns. This paper describes the major target features observed during several comet apparitions where a short duration space mission was involved.

\section{Discussion}

Our program has focused on three instrumental aims, wide-field measurement, narrow bandpass imaging (with tunable etalon filters) and spectral scanning (data cube measurements), and interferometric (Fabry-Perot Interferometers, Spatial Heterodyne Spectrometers) or integral field (image slicing or fiber fed) grating spectroscopy sensing of line profiles and line groups. The earliest observations in this program date back to comet $1973 \mathrm{f}$ (Kohoutek) (Huppler et al. 1975; Scherb 1981) and have continued through the most recent apparition of $73 \mathrm{P} / \mathrm{Sch}$ wassmann-Wachmann 3. Since then, many well known comets have been observed as part of this members of this team, most notably C/1975 V1 (West), 
1P/Halley (1986), C/1989 C1 (Austin), C/1995O1 (Hale-Bopp), C/1996B2 (Hyakutake), 9P/Tempel 1(2005), and 73P/Schwachmann-Wachman 3 (2005). While each of these was also observed by space telescopes (HST, IUE, FUSE, SOHO, etc.), three (1P/Halley, $\mathrm{C} / 199501$, and 9P/Tempel 1) were also part of short duration spacecraft encounters or space observations.

The primary diagnostic targets of this research have been Fluorescence $(\mathrm{H})$ and prompt emissions $(\mathrm{O}$ and $\mathrm{C})$ from atomic species and velocity resolved measurements of molecular ions $\left(\mathrm{H}_{2} \mathrm{O}^{+}\right)$. As end products of coma chemistry, measurements of atomic distributions provide a mass inventory of production rates for the major parent species $\left(\mathrm{H}_{2} \mathrm{O}, \mathrm{CO}\right.$, and $\mathrm{CO}_{2}$ ). Their line shapes may also carry significant non-thermal velocity signatures (e.g. Combi et al. 1999) associated with excess energy from photochemistry. Recent progress has been made in ground based observations the $\mathrm{H}_{2} \mathrm{O}$ and $\mathrm{CO}$ parents in the IR (DelloRusso et al. 2000; Crovisier and Le Bourlot 1983; Crovisier et al. 1999) and Radio (Biver et al. 2002; Neufeld et al. 2000; Lecacheux et al. 2003), however the use of daughter products as proxies for production rate continues to be a very important component of cometary research because of the complementary dynamical and distributional information they provide. Among the daughter products under study, those left in metastable states $\left(\mathrm{O}^{1} \mathrm{D}\right.$ and $\left.\mathrm{C}^{1} \mathrm{D}\right)$ also provide unique insight into the evolution of volatiles in the coma. Both $\mathrm{O}^{1} \mathrm{D}$ and $\mathrm{C}^{1} \mathrm{D}$ states have short lifetimes (115 and 4,077 s), with $\mathrm{O}^{1} \mathrm{D}$ coming from $\mathrm{H}_{2} \mathrm{O}$ and $\mathrm{OH}$ and $\mathrm{C}^{1} \mathrm{D}$ coming from $\mathrm{CO}$ (Tozzi et al. 1998). Their short lifetimes, combined with the $\mathrm{V}_{\text {outflow }} \leq 1 \mathrm{~km} / \mathrm{s}$ for most comets and the $10^{5} \mathrm{~km}$ or greater scale lengths of their primary parents, mean map of the metastable emission is effectively a map of the location of parent chemistry in the coma (Magee-Sauer et al. 1989; Morgenthaler et al. 2001; Harris et al. 2002a, b). Moreover, since a metastable atom emits only one time, the integrated flux measures the steady state mass of the parent species in the coma. This combination of factors makes it straightforward to invert the wide field brightness to production rate (Q) provided the branching ratios of the parents are understood (Schultz et al. 1992; MageeSauer et al. 1989; Oliversen et al. 2002; Morgenthaler et al. 2001, 2007). The major difficulty is that both $\mathrm{O}^{1} \mathrm{D}$ and $\mathrm{C}^{1} \mathrm{D}$ require high resolving power to separate the cometary emissions from nearby molecular bands $\left(\mathrm{NH}_{2}\right.$ and $\left.\mathrm{OH}\right)$ and from telluric emission and/or absorption features. A similar problem exists for $\mathrm{H} \alpha$ which must be Doppler-shifted off of both the geocoronal line and background galactic emission (Huppler et al. 1975; Morgenthaler et al. 2002a, b). In situ measurements can eliminate the telluric contamination, but they are limited by the lack of high resolving power instruments to separate the molecular contaminants and by the large scale of the coma in these species, especially $\mathrm{H}$ (Combi et al. 1999) in which they are embedded.

High resolving power studies of cometary ion lines are also important for understanding both the coma and solar wind environments. Provided the production rates are known for the ion parents (typically $\mathrm{H}_{2} \mathrm{O}^{+}$or $\mathrm{CO}^{+}$), the distribution of ions and the evolution of their velocity distribution as they move back into the tail can be tied directly to the density and velocity of the local solar wind. Since a single comet apparition will sample a large volume of the inner solar system, including regions off the plane of the ecliptic, they are thus 'wind-socks' sampling space weather in area where we have no capability for doing so ourselves. The role of resolving power in this analysis is that mass loading of the solar wind increases cometary ion velocities in the inner coma from the initial combination of the nucleus Keplerian velocity $(\sim 10-100 \mathrm{~km} / \mathrm{s})$ and expansion velocity $(\sim 1 \mathrm{~km} / \mathrm{s})$ of the coma to a momentum balance with the $(\sim 100-1,000 \mathrm{~km} / \mathrm{s})$ anti-sunward velocity of the solar wind. At high resolution this acceleration can be mapped with location in the coma 
and tail using wavelength stepped interferometric imaging (data cubes) or wide field integral field spectroscopy (Anderson 1999).

\section{Observational Synergy: Results from C/199501 (Hale-Bopp)}

The 1997 apparition of c/1995OI (Hale-Bopp) provides an excellent illustrative example of the how a ground based diagnostic suite may be combined synergistically with space-based observations to enhance the overall scientific return of both sets. Hale-Bopp was not visited by an in situ spacecraft, but was observed by a series of sub-orbital sounding rocket experiments, the SOHO spacecraft, and the Hubble Space Telescope (HST). The very high activity (peak $\mathrm{Q}_{\mathrm{H}_{2} \mathrm{O}}>10^{31} \mathrm{~s}^{-1}$ ) permitted a full sampling of all aspects of the coma our ground-based program can currently access, and it therefore is the best example of what synergy is possible. Sounding rocket wide-field imaging polarimetry (Harris et al. 1999) obtained images of the full coma of atomic carbon from resonance scattering of the CI ( $\lambda 1657)$ multiplet that were inverted directly back to a total carbon production rate. A second rocket experiment (McPhate et al. 1998) obtained narrow slit spectroscopy of both CI ( 21657$)$ and fourth positive band of CO in the inner coma. The latter observations are invertible from radial distributions to production rates of each species provided the outflow velocity of the coma is known. Similar wide field measurements of scattered solar H Ly- $\alpha$ in the coma (Combi et al. 2000) with SOHO provide a total hydrogen (effectively water) production rate outside of the optically thick inner coma. (While this was not done for Hale-Bopp, line-shape measurements with HST (Combi et al. 1999) further sample the complex non-thermal line shape of the Ly- $\alpha$ line.) In addition, the trio of $\mathrm{CO}, \mathrm{CO}_{2}$, and $\mathrm{H}_{2} \mathrm{O}$ were observed in the inner coma by ISO (Crovisier et al. 1999).

In support of these efforts a large, multi-observatory campaign was mounted close in time to the sounding rocket campaign near perihelion, during which wide field $\mathrm{H} L \mathrm{~L}-\alpha$ images were also obtained (Combi et al. 2000). A full series of observational diagnostics were obtained from small $(0.9 \mathrm{~m}$ Burrell Schmidt) to intermediate $(3.5 \mathrm{~m}$ WIYN) telescopes that included interferometric imaging of $\mathrm{O}^{1} \mathrm{D}$ (Fig. 1) and $\mathrm{H}_{2} \mathrm{O}^{+}$(Fig. 2), high resolving power measurements of $\mathrm{O}^{1} \mathrm{D}, \mathrm{C}^{1} \mathrm{D}$ (Fig. 3), $\mathrm{H} \alpha$ (Fig. 4), and $\mathrm{H}_{2} \mathrm{O}^{+}$(Fig. 5), and narrow band imaging of $\mathrm{OH}$ (Fig. 6), $\mathrm{CN}, \mathrm{C}_{3}$ and $\mathrm{C}_{2}$. The results of these observations provide important supplemental context for understanding the space-based data.

\subsection{Carbon Observations}

The $C^{1} \mathrm{D}$ wide field observations (Oliversen et al. 2002, Fig. 3) essentially measured the photo-destruction of $\mathrm{CO}$ in the coma, since $\mathrm{CO}_{2}$ has a minimal branching ratio to this reaction (Huebner et al. 1992). It therefore provided a direct measure of its parent over a slightly larger FOV than the rocket CO observation (McPhate et al. 1998) and a comparison of $\mathrm{Q}_{\mathrm{CO}}$ with $\mathrm{Q}_{\mathrm{C}}$ as observed with the wide-field imaging rocket (Harris et al. 1999) and with $\mathrm{Q}_{\mathrm{H}_{2} \mathrm{O}}$ (via $\mathrm{H} \mathrm{Ly}-\alpha$ from SOHO and $\mathrm{H} \alpha$ from ground based measurement).

\subsection{Water Daughter Products and Coma Dynamics}

Direct full coma measurements of H Ly- $\alpha$ may, in principle, be inverted to water production provided opacity effects are addressed and the velocity components are addressed. Hydrogen's velocity distribution is highly non-thermal due to the large amounts of excess energy pumped into it from the photodissociation of its parents. The accuracy of the 


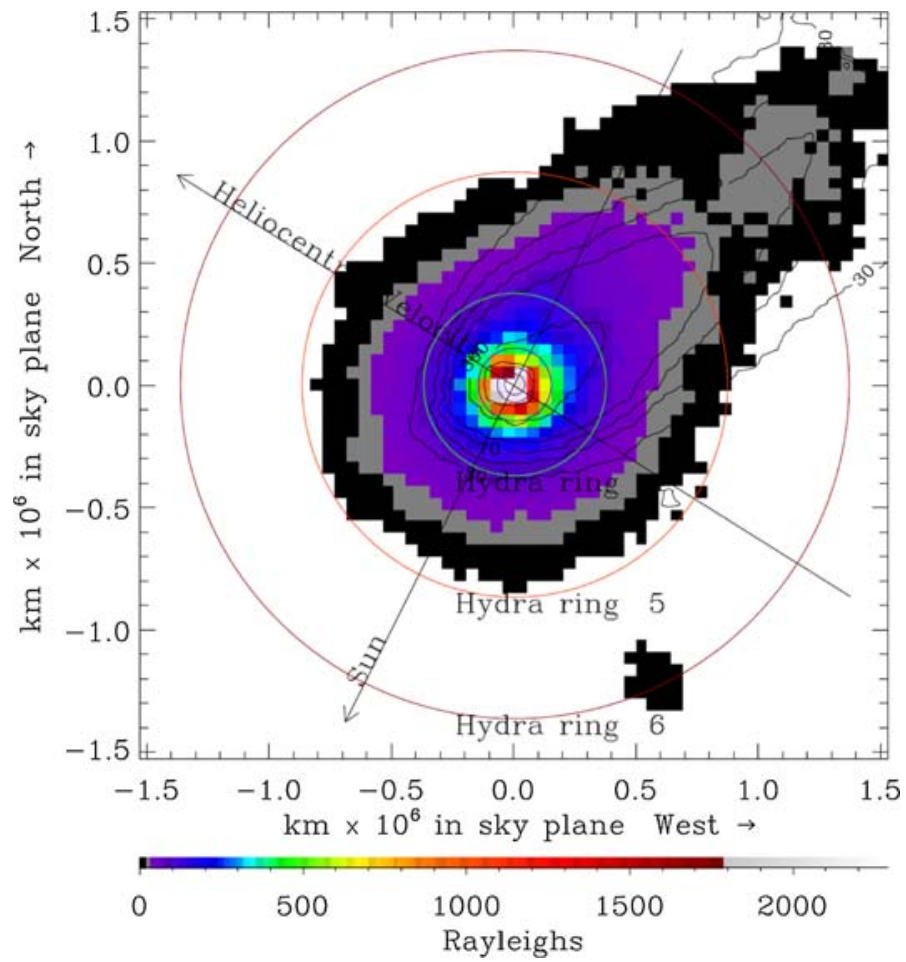

Fig. 1 A narrow band image of $\mathrm{O}^{1} \mathrm{D}$ emission from comet C/1995 O1 (Hale-Bopp) take with the Wisconsin $\mathrm{H}-\alpha$ Mapper (WHAM), a stand-alone Fabry-Perot interferometer operated by the University of Wisconsin on Kitt Peak (from Morgenthaler et al. 2002a). Source: Harris et al. 2002a (Earth, Moon, and Planets Vol 90)
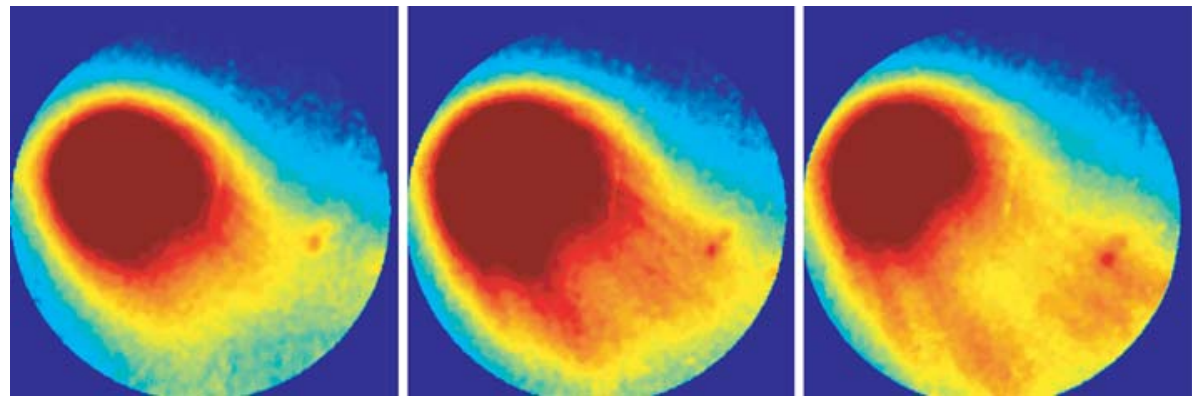

Fig. 2 A series of narrow band images from a data-cube of $\mathrm{H}_{2} \mathrm{O}^{+}$emission from comet $\mathrm{C} / 1995 \mathrm{O} 1$ (HaleBopp) shows acceleration into the tail. Each image is $<10 \mathrm{~km} / \mathrm{s}$ step in velocity

inversion is dependent on an understanding of the photochemical branching ratios leading to $\mathrm{H}$ from $\mathrm{H}_{2} \mathrm{O}$ and on the importance of collisions within the photochemical scale length (Combi et al. 2000). Ground based observations of $\mathrm{OH}$ (Harris et al. 2002a, b), $\mathrm{O}^{1} \mathrm{D}$ (Morgenthaler et al. 2001, Morgenthaler et al. 2007), and $\mathrm{H} \alpha$ (Morgenthaler et al. 2002a, b) all reveal a complex environment with implications for estimates of $\mathrm{Q}_{\mathrm{H}_{2} \mathrm{O}}$. Modeling of the radial distribution of $\mathrm{OH}$ showed significant evidence of thermalization of photochemical 


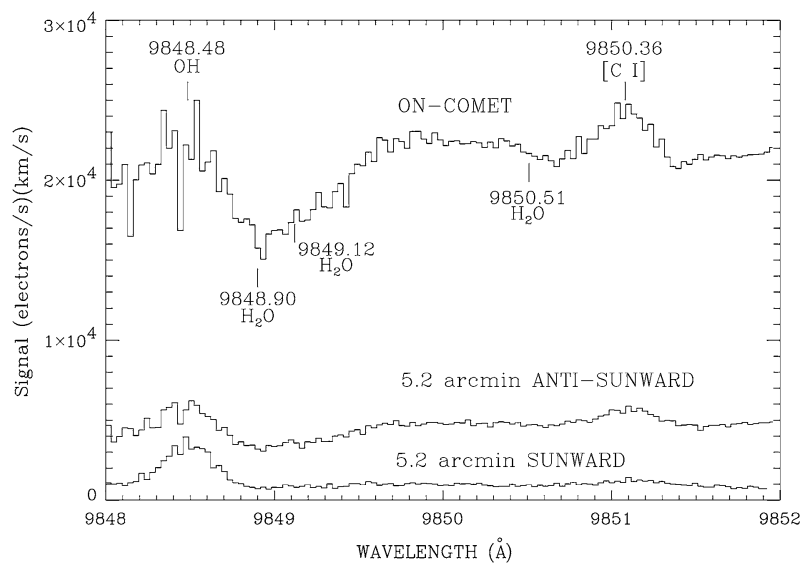

Fig. 3 A spectrum of $C^{1} \mathrm{D}$ emission from comet C/1995 O1 (Hale-Bopp) shows the carbon emission along side contaminating $\mathrm{OH}$ and $\mathrm{H}_{2} \mathrm{O}$ telluric features (from Oliversen et al. 2002. Reproduced by permission of the AAS)

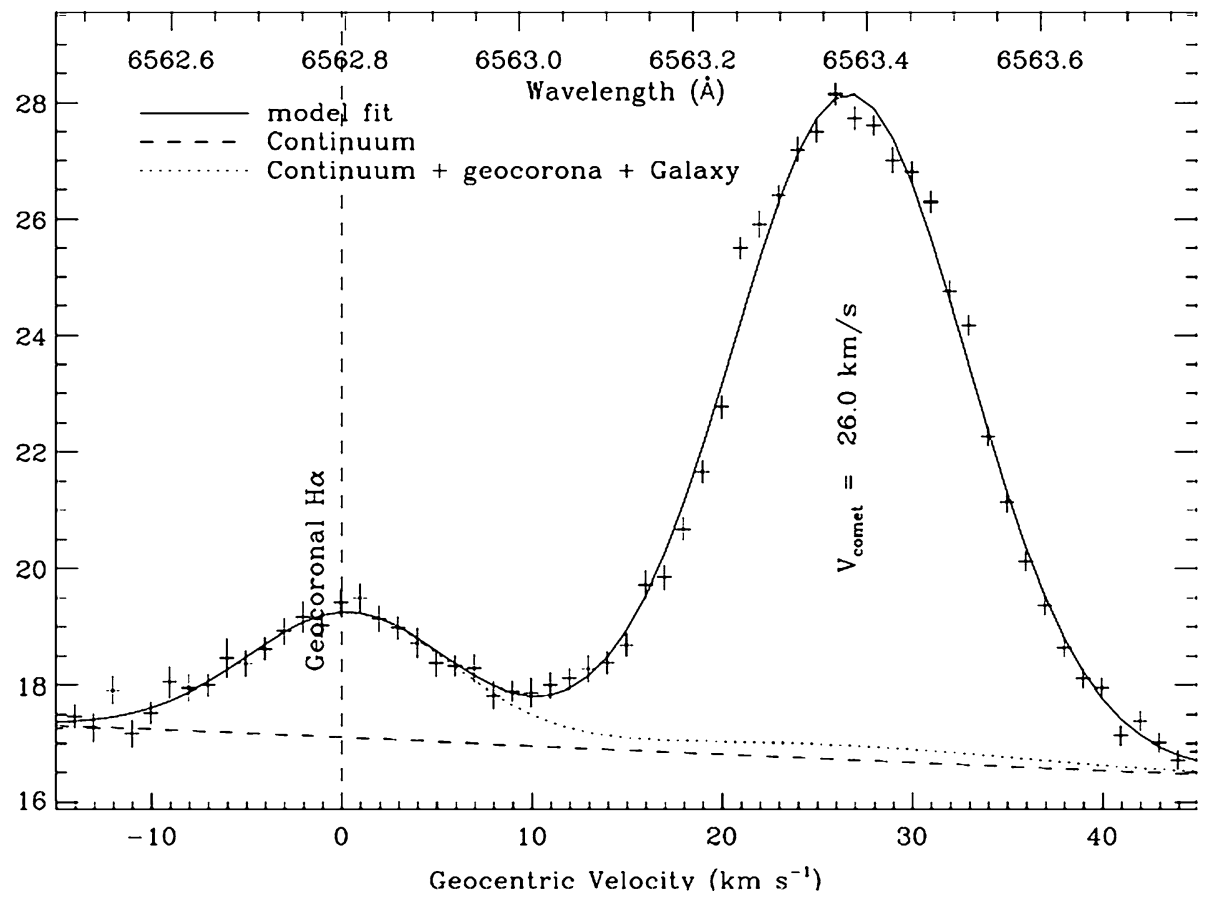

Fig. 4 A spectrum of $\mathrm{H} \alpha$ emission from comet C/1995 O1 (Hale-Bopp) obtained with the WHAM instrument. The spectrum shows both the comet and geocoronal lines and demonstrates the significance of using high resolving power and the Doppler shift of the comet to obtain a clean signal (from Morgenthaler et al. 2002b). Source: Morgenthaler et al. 2000 (Earth, Moon, and Planets Vol 90) 
Hale-Bopp 40" ring 1996 Oct. 22 WIYN/Hydra/MOS

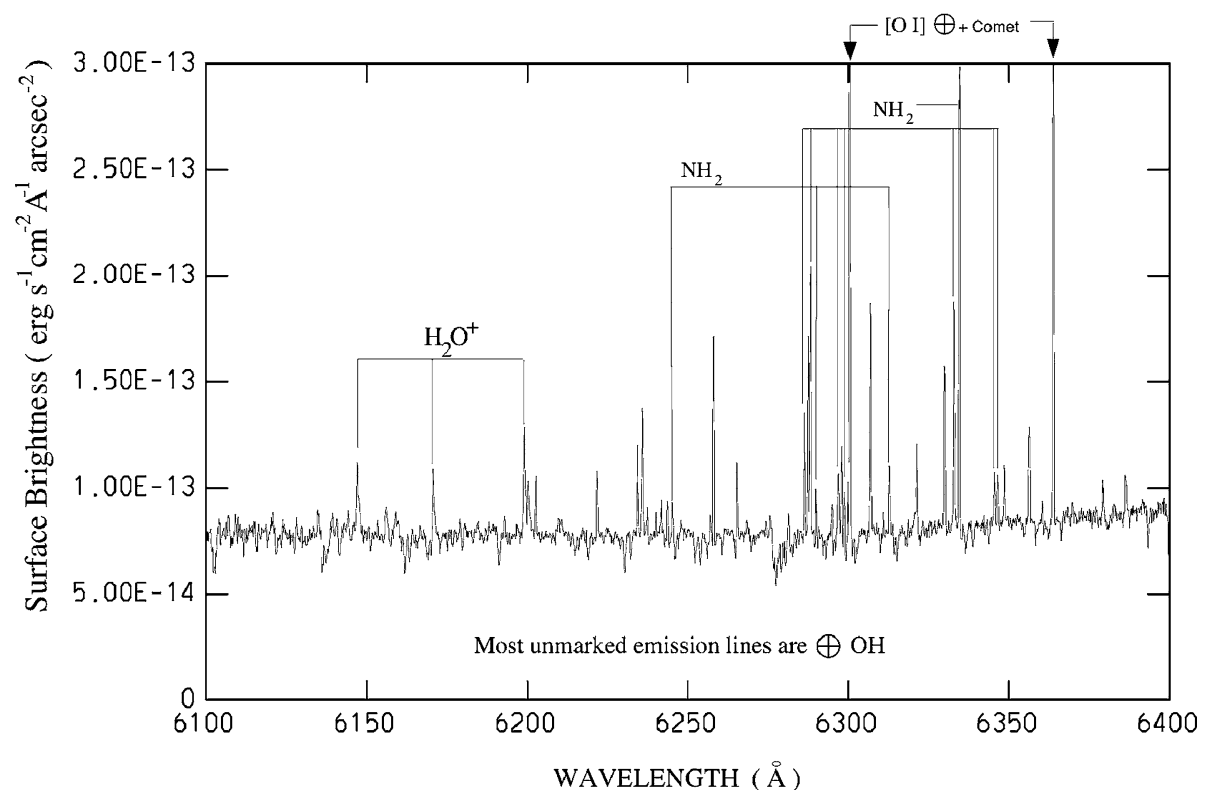

Fig. 5 An echelle spectrum extracted from a single fiber (of 100) positioned 40" from the nucleus of comet C/1995 O1 (Hale-Bopp) using the Hydra integral field spectrometer at the WIYN telescope shows comet features from $\mathrm{H}_{2} \mathrm{O}^{+}, \mathrm{NH}_{2}$, and $\mathrm{O}^{1} \mathrm{D}$ with a centroid velocity resolution of $2-3 \mathrm{~km} / \mathrm{s}$ (image courtesy C. M. Anderson). The Hydra fibers were positioned in a series of concentric rings extending out to 20 arcminutes from the nucleus. Source: Anderson 1999 (Earth, Moon, and Planets Vol. 78)

excess energy leading to an increase in the radial expansion rate of the coma (Harris et al. $2002 \mathrm{a}, \mathrm{b})$. This result was confirmed using radial maps of $\mathrm{O}^{1} \mathrm{D}$ that showed evidence of deviation from published photochemical branching ratios (Morgenthaler et al. 2001, 2007). $\mathrm{H} \alpha$ line shape analysis showed broadening consistent with the expected $\mathrm{H}$ outflow characteristics in the outer coma, but also for substantial optical depth effects in the inner regions (Morgenthaler et al. 2002b). This combination of multiple daughters observed both spatially and in Doppler space produced a far richer understanding of the coma and water.

\subsection{Ion Studies}

With its high orbital inclination Hale-Bopp sampled a range of high solar latitude regions that are difficult to observe with existing spacecraft such as SOHO or Ulysses. Both imaging and data cube observations of $\mathrm{H}_{2} \mathrm{O}^{+}$(Figs. 2 and 5) reveal clear evidence of acceleration in the anti-sunward direction. However, the rate of acceleration is substantially less than was measured from 1P/Halley in 1986 (Scherb et al. 1990; Anderson 1999). While the combination of a different solar wind environment and the increased mass of gas production from Hale-Bopp relative to $1 \mathrm{P} /$ Halley may have led to a significant decrease in the inner coma acceleration of the cometary ions, images of $\mathrm{OH}$ and $\mathrm{O}^{1} \mathrm{D}$ emission paint a different story. In both cases there is clear evidence of an emission enhancement in a direction between the anti-sunward and anti-orbital axes of the comet orbit. As part of their broader treatment of these data (Harris et al. 2002a, b; Morgenthaler et al. 2001) this 


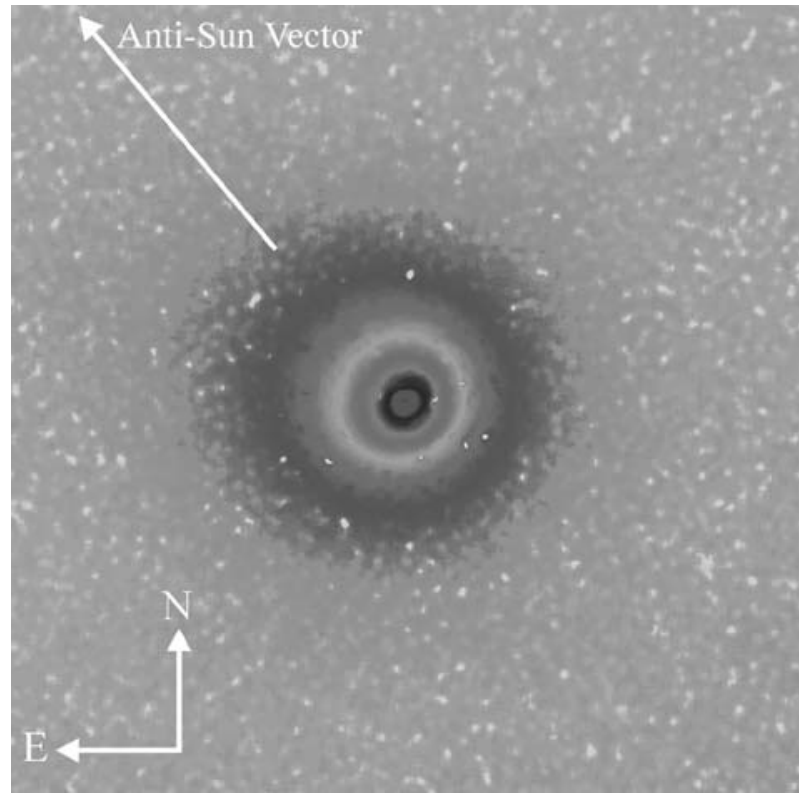

Fig. 6 A narrow band image of $\mathrm{OH}$ obtained with the Burrell Schmidt telescope on Kitt Peak shows a slightly asymmetric coma with the extension aligned toward the antisunward direction (from Harris et al. 2002b. Reproduced by permission of the AAS). Source: Harris et al. 2002 (Ap. J. Vol 576)

deflection was attributed to possible ion-neutral collisions (Figs. 1 and 6) in the thick regions of the coma that extended to more than $10^{5} \mathrm{~km}$ from the nucleus (Harris et al. $2002 \mathrm{~b}$ ). This result fit well with the conclusion, based on the coma scale length of $\mathrm{OH}$, that Hydrogen photochemical excess energy was being re-thermalized into the bulk coma outflow in Hale-Bopp. It also implies that comets with very high water production rates may be less diagnostic of the local solar wind characteristics, or at least require a collisional component in the analysis.

\section{Conclusions}

Space based observational studies of comets are well supported by supplemental ground based campaigns focusing on providing complementary instrumental capabilities, timing, and perspective. High spectral resolution instruments at small to medium aperture telescopes are more than adequate for providing this capability for active comet apparitions, with the additional benefit that precise scheduling and/or long duration campaigns are possible. Results from the 1997 Hale-Bopp perihelion campaign showed the value obtained with ground and space observatories are used collaboratively and that smaller telescope facilities can contribute significantly to the overall science return. Future comet encounter missions should benefit from future programs.

Open Access This article is distributed under the terms of the Creative Commons Attribution Noncommercial License which permits any noncommercial use, distribution, and reproduction in any medium, provided the original author(s) and source are credited. 


\section{References}

C. Anderson, Earth Moon Planet 78, 99-104 (1999)

N. Biver et al., Earth Moon Planet 90, 5-14 (2002)

M.R. Combi, A.A. Reinard, J.-L. Bertaux, E. Quemerais, T. Mäkinen, Icarus 144, 191 (2000)

M.R. Combi, A.L. Cochran, W.D. Cochran, D.L. Lambert, C.M. Johns-Krull, Ap. J. 512, 961-968 (1999)

J. Crovisier, K. Leech, D. Bockelée-Morvan, E. Lellouch, T. Y. Brooke, M. S. Hanner, B. Altieri, H. U. Keller, T. Lim, The Universe as Seen by ISO ESA SP-427, 137-140 (1999)

J. Crovisier, J. Le Bourlot, Astron. Astrophys. 123, 61-66 (1983)

N. DelloRusso, M.J. Mumma, M.A. DiSanti, K. Magee-Sauer, Icarus 143, 324-337 (2000)

W.M. Harris, K.H. Nordsieck, F. Scherb, E.J. Mierkiewicz, Earth Moon Planet 78, 161 (1999)

W.M. Harris, J.P. Morgenthaler, F. Scherb, C. Anderson, R.J. Oliversen, Earth Moon Planet 90, 45 (2002a)

W.M. Harris, F. Scherb, E.J. Mierkiewicz, R.J. Oliversen, J.P. Morgenthaler, Ap. J. 578, 996 (2002b)

W.F. Huebner, J.J. Keady, S.P. Lyon, Astrophys. Space Sci. 195, 7 (1992)

D. Huppler, R.J. Reynolds, F.L. Roesler, F. Scherb, J. Trauger, Astrophys. Space Sci. 195, 1-294 (1975)

A. Lecacheux et al., Astron. Astrophys. 402, L55-L58 (2003)

K. Magee-Sauer, F. Scherb, F.L. Roesler, J. Harlander, B.L. Lutz, Icarus 82, 50-60 (1989)

J.B. McPhate, P.D. Feldman, S.R. McCandliss, E.B. Burgh, Ap. J. 521, 920 (1998)

K. Meech et al., Science 310, 265-269 (2005)

J.P. Morgenthaler, W.M. Harris, F. Scherb, C. Anderson, R.J. Oliversen, N.E. Doane, M.R. Combi, M.L. Marconi, W.H. Smyth, Ap. J. 563, 451-461 (2001)

J.P. Morgenthaler, W.M. Harris, F. Scherb, N.E. Donae, R.J. Oliversen, Earth Moon Planet 90, 77-87 (2002a)

J.P. Morgenthaler, F.L.,W.M. Harris Roesler, F. Scherb, C.M. Anderson, N.E. Donae, R.J. Oliversen, Earth Moon Planet 90, 89-97 (2002b)

J.P. Morgenthaler, W.M. Harris, M.R. Combi, Ap. J. 657, 1162-1171 (2007)

D.A. Neufeld et al., Ap. J. Lett. 539, L151-L154 (2000)

R.J. Oliversen, N. Doane, F. Scherb, W.M. Harris, J.P. Morgenthaler, Ap. J. 581, 770-775 (2002)

F. Scherb, Ap. J. 243, 644-650 (1981)

F. Scherb, K. Magee-Sauer, F.L. Roesler, J. Harlander, Icarus 86, 172-188 (1990)

D. Schultz, G.S.H. Li, F. Scherb, F.L. Roesler, Icarus 96, 190 (1992)

G.P. Tozzi, P.D. Feldman, M.C. Festou, Astron. Astrophys. 330, 753 (1998) 